\title{
Form and function of the condensed bacterial nucleoid studied by cryo-ET
}

\author{
Daniel Parrell ${ }^{1}$, Joseph Olson ${ }^{2}$, Timothy Donohue ${ }^{3}$ and Elizabeth Wright ${ }^{4}$
}

${ }^{1}$ Department of Biochemistry, University of Wisconsin - Madison, United States, ${ }^{2}$ Department of Biochemistry, University of Wisconsin, Illinois, United States, ${ }^{3}$ Department of Bacteriology, University of Wisconsin, Madison, Wisconsin, United States, ${ }^{4}$ Department of Biochemistry, University of WisconsinMadison, Madison, Wisconsin, United States

All cells share a common genetic code. From the nucleus of mammalian cells to the nucleoid of the smallest bacterium, all cells use DNA to encode the traits and cellular processes that drive the diversity of life on earth. Replication of the genome is the most fundamental form of heritability and errors or damage during this process can have dire consequences. It is therefore of great importance that all organisms carry out mechanisms to protect nuclear material in the event of cellular stress. To study the form and function of DNA structures during cellular stress, we are using cryo-electron tomography (cryo-ET) of bacteria. In doing this, we use the bacterial nucleoid as a reporter of in vivo chromosome structure, and to study the replication and protection of a cells' genetic material.

For this work we are using Rhodobactersphaeroides, a facultative alphaproteobacterium with features that make it attractive for analysis. R. sphaeroides cultures were grown at $30^{\circ} \mathrm{C}$ and $200 \mathrm{RPM}$ shaking in Sistrom's minimal medium until the culture reached an $\mathrm{OD}_{600}$ of 0.4. Cells were then treated with the antibiotic chloramphenicol or transferred to ice to produce cold shock. Suspensions of the cells were deposited onto 200 mesh R2/1 copper Quantifoil grids in $5 \mu \mathrm{L}$ aliquots, blotted, and plunge frozen in liquid ethane using a Vitrobot Mark IV (ThermoScientific). Cryo-electron microscopy (cryo-EM) and cryo-electron tomography (cryo-ET) data were collected using a Titan Krios TEM (ThermoScientific) operated at $300 \mathrm{kV}$, equipped with a Bioquantum energy filter and a K3 direct electron detector (Gatan). Single axis tilt series were acquired using SerialEM (1), with an increment of $2^{\circ}$ covering $-60^{\circ}$ to $+60^{\circ}$ and a cumulative dose under $150 \mathrm{e}^{-} / \AA^{2}$ at a defocus range between -4 and $-10 \mu \mathrm{m}$. Tomograms were reconstructed using IMOD/eTomo (2), and 3D rendering was performed using EMAN2 neural network segmentation training(3).

This work seeks to observe and compare the structure and interactions of the nucleoid during normal replication and in response to a cellular stress such as cold shock or antibiotic treatment. Upon cold shock a condensed nucleoid is observed by cryo-ET (Figure 1). Repeating striations that swirl through the cell are representative of nuclear material. Observations on the spacing of the DNA strands under cold shock, and modeling of the nucleoid within the cell and in conjunction with other cellular structures such as vesicles, ribosomes and storage granules will also presented based on segmentation analyses. Cryo-ET studies also reveal a condensed nucleoid upon treatment with the protein synthesis inhibitor chloramphenicol (Figure 2). Additionally, stressinduced changes to cell structure observed by cryo-ET, including changes to cell size and accumulation of storage materials will also be presented. These observations illustrate the ability of cryo-ET to monitor the cellular effects of stress and antibiotic treatment. Future work will be targeted to understand mechanisms of nucleoid condensation and the function of condensed DNA for survival and persistence of cells during stress. This work seeks gain a better understanding of the mechanisms bacteria use to survive and persist during cold shock stress and during antibiotic treatment. 

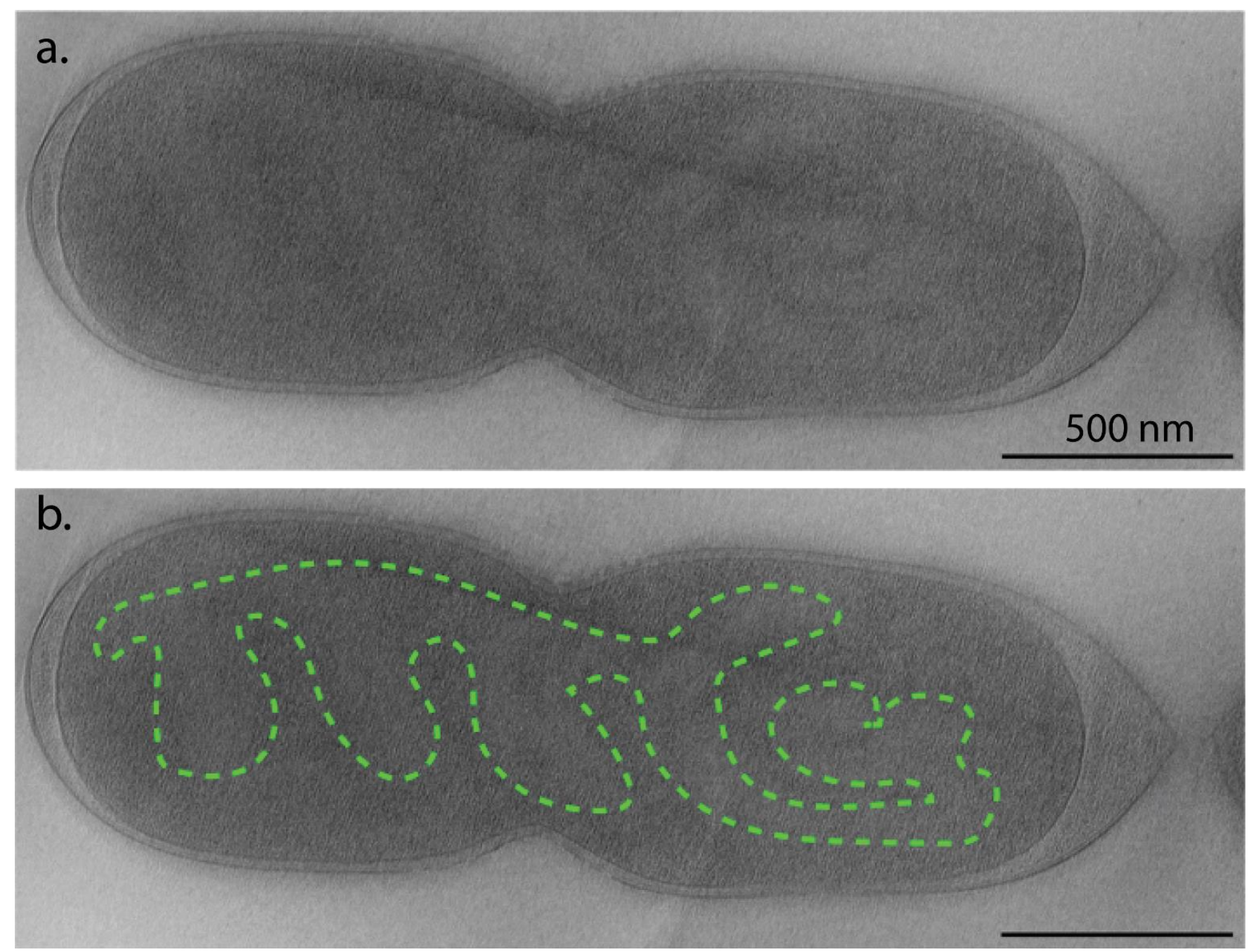

Figure 1. Figure 1. a. A slice through the reconstructed tomogram of a cell demonstrating condensed nuclear material during cell division. b. The same slice with an outline demonstrating the location of condensed nucleoid. Scale is $500 \mathrm{~nm}$. 
No treatment

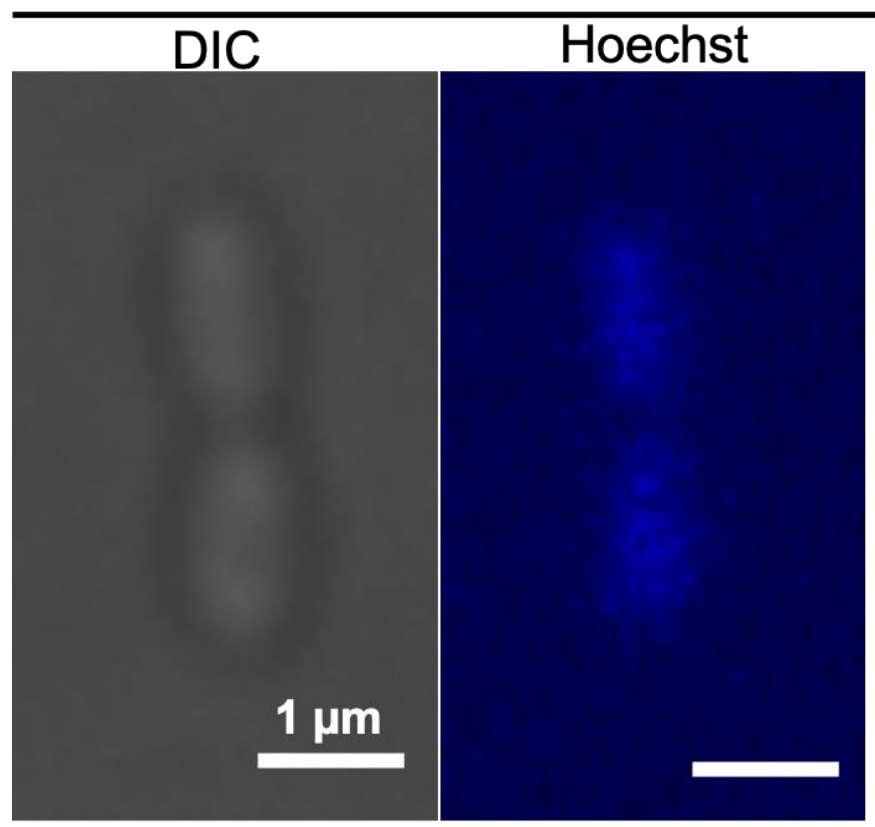

$200 \mu \mathrm{g} / \mathrm{mL}$ Chloramphenicol

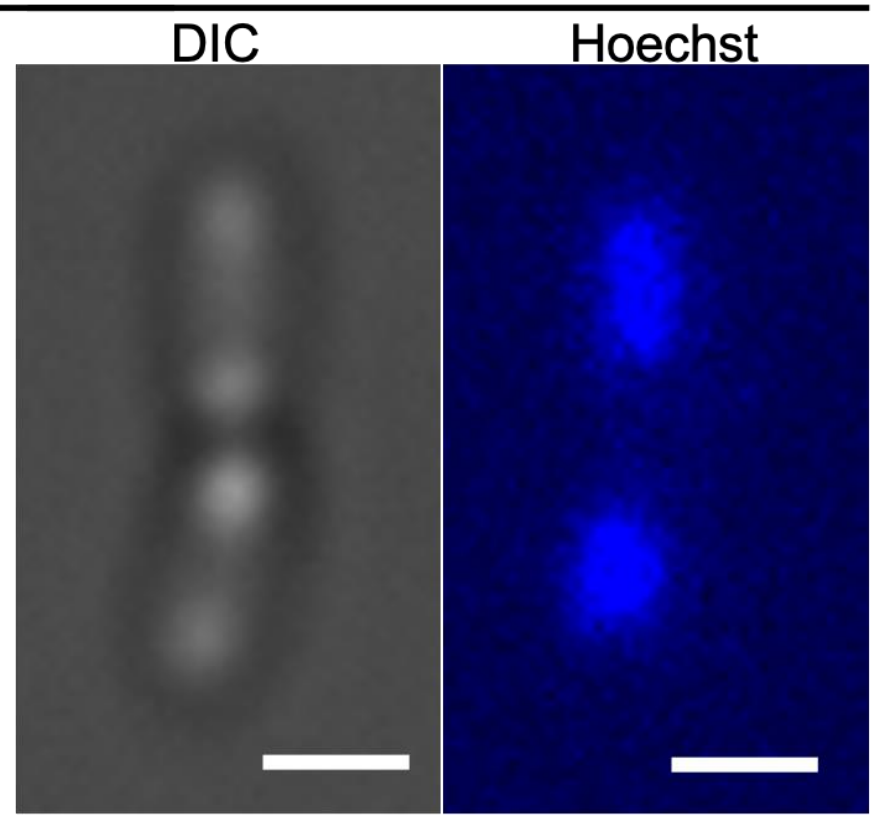

Figure 2. Figure 2. Nucleoid compaction in R. sphaeriodes. Cells grown to OD600 of 0.4 were allowed to continue incubation ( $\mathrm{a} \& \mathrm{~b}$ ) or were treated with $\mathrm{Cm}$ (c \& d). Bright nuclear foci stained by Hoechst DNA stain (d) and tight localization within the cells DIC image (c) represents evidence of nucleoid compaction relative to no treatment.

References

1. Mastronarde DN. Automated electron microscope tomography using robust prediction of specimen movements. J Struct Biol. 2005;152(1):36-51. Epub 2005/09/27. doi: 10.1016/j.jsb.2005.07.007. PubMed PMID: 16182563.

2. Kremer JR, Mastronarde DN, McIntosh JR. Computer visualization of three-dimensional image data using IMOD. J Struct Biol. 1996;116(1):71-6. Epub 1996/01/01. doi: 10.1006/jsbi.1996.0013. PubMed PMID: 8742726.

3. Chen M, Dai W, Sun SY, Jonasch D, He CY, Schmid MF, Chiu W, Ludtke SJ. Convolutional neural networks for automated annotation of cellular cryo-electron tomograms. Nat Methods. 2017;14(10):983-5. Epub 2017/08/29. doi: 10.1038/nmeth.4405. PubMed PMID: 28846087; PMCID: PMC5623144.

4. This research was supported by funds from the University of Wisconsin-Madison, National Institutes of Health (R01GM104540 and R01GM104540-03S1) to E.R.W, and the Great Lakes Bioenergy Research Center (DOE DE-SC0018409) to T.D. All EM data was collected at the University of Wisconsin-Madison, Department of Biochemistry Cryo-EM Research Center. 